

\title{
Prediction of Borderzone Infarction by CTA in Patients Undergoing Carotid Embolization for Carotid Blowout
}

\author{
(D).-C. Lee, (D).-H. Lin, (D).-W. Lee, (D) H.-M. Liu, and (D) A. Huang
}

\begin{abstract}
BACKGROUND AND PURPOSE: Permanent common carotid artery and/or ICA occlusion is an effective treatment for carotid blowout syndrome. Besides postoperative thromboembolic infarction, permanent common carotid artery and/or ICA occlusion may cause borderzone infarction when the collateral flow to the deprived brain territory is inadequate. In this study, we aimed to test the predictive value of CTA for post-permanent common carotid artery and/or ICA occlusion borderzone infarction in patients with carotid blowout syndrome.
\end{abstract}

MATERIALS AND METHODS: In this retrospective study, we included 31 patients undergoing unilateral permanent common carotid artery and/or ICA occlusion for carotid blowout syndrome between May 2009 and December 2016. The vascular diameter of the circle of Willis was evaluated using preprocedural CTA, and the risk of borderzone infarction was graded as very high risk, high risk, intermediate risk, low risk, and very low risk.

RESULTS: The performance of readers' consensus on CTA for predicting borderzone infarction was excellent, with an area under receiver operating characteristic curve of 0.938 (95\% confidence interval, $0.85-1.00)$. We defined very high risk, high risk, and intermediate risk as positive for borderzone infarction, the sensitivity, specificity, positive predictive value, and negative predictive value of CTA for borderzone infarction were 100\% (7/7), 62.5\% (15/24), 43.8\% (7/16), and 100\% (15/15), respectively. The interobserver reliability was excellent $(\kappa=$ 0.807). No significant difference in the receiver operating characteristic curves was found between the 2 readers $(P=.114)$.

CONCLUSIONS: CTA can be used to predict borderzone infarction after permanent common carotid artery and/or ICA occlusion by measuring the collateral vessels of the circle of Willis.

ABBREVIATIONS: AcomA = anterior communicating artery; $\mathrm{A}_{\mathrm{z}}=$ area under the ROC curve; $\mathrm{BTO}=$ balloon test occlusion; $\mathrm{CBS}=$ carotid blowout syndrome; $\mathrm{CCA}=$ common carotid artery; $\mathrm{PCO}=$ permanent common carotid artery and/or ICA occlusion; $\mathrm{PcomA}=$ posterior communicating artery; $\mathrm{ROC}=$ receiver operating characteristic

$\mathbf{R}^{\mathrm{n}}$ upture of the carotid artery and its branches, referred to as carotid blowout syndrome (CBS), is an infrequent but dreaded complication of head and neck cancer, which occurs in up to $4.3 \%$ of patients. ${ }^{1-4}$ Patients with head and neck cancer who have radiation-induced necrosis, tumor recurrence, pharyngocu-

Received December 18, 2017; accepted after revision March 24, 2018.

From the Department of Medical Imaging (B.-C.L., Y.-H.L., C.-W.L.), National Taiwan University Hospital and National Taiwan University College of Medicine, Taipei, Taiwan; Department of Radiology (H.-M.L.), Fu-Jen Catholic University Hospital, New Taipei, Taiwan; and Research Center for Adaptive Data Analysis (A.H.), National Central University, Jhongli, Taiwan.

This work was supported by the Ministry of Science and Technology (MOST 1062218-E002-018) and the Ministry of Health and Welfare (MOHW107-TDU-B-212123004) of Taiwan.

Please address correspondence to Chung-Wei Lee, MD, PhD, Department of Medical Imaging, National Taiwan University Hospital, No. 7, Chung-Shan South Rd, Taipei 100, Taiwan, R.O.C.; e-mail: rad.chungweilee@gmail.com

O-- Indicates open access to non-subscribers at www.ajnr.org

EIndicates article with supplemental on-line tables.

http://dx.doi.org/10.3174/ajnr.A5672 taneous fistulas, or wound complications are more prone to develop CBS. ${ }^{5,6}$ The emergency surgical management of CBS, which involves ligating the common carotid artery (CCA) or ICA, is a technically challenging procedure that is associated with high risk of cerebral ischemia. ${ }^{4,7,8}$ For a patient with CBS and a diseased CCA and/or ICA, permanent CCA and/or ICA occlusion (PCO) with coils is an effective treatment that produces better clinical outcomes compared with stent-graft placement ${ }^{9}$ and has been the standard treatment in our institution. Still, thromboembolic infarction and borderzone infarction occurred in approximately $20 \%$ of patients. ${ }^{9}$ Balloon test occlusion (BTO) is the optimal testing method that can simulate the collateral reserve of the circle of Willis, which is associated with hypoperfusion-related borderzone infarction after PCO. ${ }^{10-12}$ However, the suitability of BTO during emergent $\mathrm{PCO}$ for CBS is contentious, and the complication rate of BTO has been reported to be as high as 3.2\%, which might be even higher for patients with CBS in whom heparin infusion is contraindicated. ${ }^{12,13}$ 
Because of the superior ability of CTA to detect an exposed artery compared with angiography, CTA has become a valuable tool for procedure planning in patients with CBS. ${ }^{14}$ The diameter of the collateral vessels in the circle of Willis can be measured on CTA using thin-sliced images with MIP and using MPR techniques. ${ }^{15}$ Therefore, we hypothesized that CTA can stratify the cerebral collateral reserve of the circle of Willis, permitting prediction of borderzone infarction. In this study, we aimed to estimate the predictive value of CTA for post-PCO borderzone infarction in patients with CBS.

\section{MATERIALS AND METHODS}

This retrospective study was approved by the institutional review board of National Taiwan University Hospital, and the requirement for informed consent was waived. The medical records of patients with CBS treated with PCO between May 2009 and March 2016 were retrieved and reviewed. The inclusion criteria were patients with a history of head and neck cancer, treated with an operation and/or chemotherapy and/or radiation therapy, having undergone unilateral PCO following CBS. There were 42 patients identified; 11 of them were excluded because of contralateral ICA occlusion $(n=1)$, ipsilateral extracranial intracranial bypass $(n=1)$, spontaneous ipsilateral ICA thrombosis after local compression $(n=1)$, prolonged ( $>5$ minutes) cardiovascular resuscitation before the procedure $(n=1)$, no preprocedural CTA of the circle of Willis due to a clinical emergency $(n=3)$, and no available thin-slice ( $\leq 1 \mathrm{~mm}$ ) CTA images for detailed evaluation of the circle of Willis $(n=4)$. No patient was identified as having a contralateral ICA, bilateral vertebral arteries, or basilar artery stenosis $(>50 \%)$. Finally, 31 patients were enrolled in our study.

\section{CTA Imaging Protocol}

The routine CTA protocol for CBS was as previously reported. ${ }^{14}$ In brief, a 64-detector row CT scanner (LightSpeed VCT; GE Healthcare, Milwaukee, Wisconsin) was used for CTA imaging, with 3 scanning phases (precontrast, arterial, and venous). The CTA protocol was as follows: $64 \times 0.625$ collimation, 0.516 pitch, 0.4 -second rotation time, and $100 \mathrm{kV}$ (peak). We used $200 \mathrm{~mA}$ for the precontrast phase and $400 \mathrm{~mA}$ for the arterial and venous phases. Dual injectors were used. The scanning range was between the aortic arch and the level of the lateral ventricles, and the timing of CTA was determined by the test-bolus technique. At a rate of 4 $\mathrm{mL} / \mathrm{s}$, the contrast material $(60 \mathrm{~mL})$ and a saline chaser $(35 \mathrm{~mL})$ were injected after a delay to generate the arterial phase, and the venous phase was obtained after another 30 seconds. The source images were reconstructed into 0.625 - or 1-mm slice thicknesses in axial, coronal, and sagittal views.

\section{Visibility of the Circle of Willis}

The CTA images were evaluated for the following segments of the circle of Willis: the A1 and A2 segments of the anterior cerebral artery, the anterior communicating artery (AcomA), the P1 and $\mathrm{P} 2$ segments of the posterior cerebral artery, and the posterior communicating artery (PcomA). One radiologist (B.-C.L.) who did not participate in the evaluation of the circle of Willis graded the quality of CTA in 3 categories: excellent, indicating high-qual-

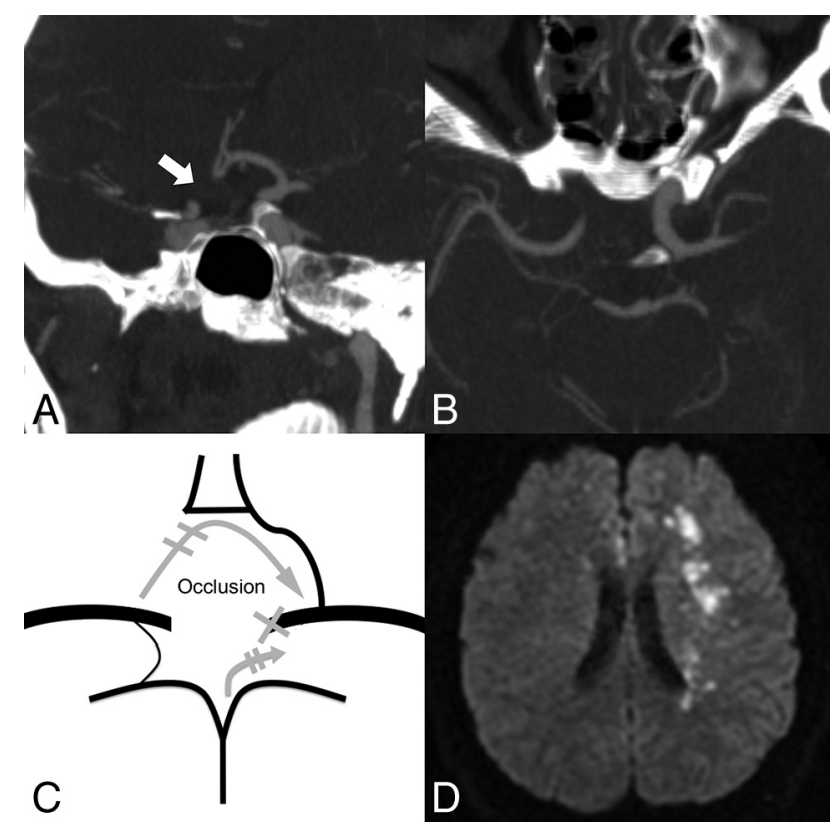

FIG 1. A 33-year-old man with nasopharyngeal carcinoma and left ICA blowout. $A$, No right $\mathrm{Al}$ segment is detected on the coronal MIP (5 $\mathrm{mm}$ ) image (white arrow). B. Only a hair-thin PcomA is detected on the axial MIP $(5 \mathrm{~mm})$ image. $C$, The narrowest portion of the anterior and posterior collateral vessels is aplastic, and the patient is grouped as very high risk. The recruited collateral flows after PCO are indicated by gray arrows. D, Diffusion-weighted imaging shows left borderzone infarction and bilateral anterior cerebral artery thromboembolic infarction on the same day after left ICA embolization.

ity delineation of the circle of Willis; fair, indicating slightly lower quality, but still useful for delineation of the circle of Willis; and poor, indicating suboptimal for evaluation of the circle of Willis.

\section{Evaluation and Data Analysis}

All CTA images (source images plus MIP images) were evaluated independently by 2 attending neuroradiologists (C.-W.L. and Y.-H.L. with 11 and 4 years of experience, respectively), blinded to patient identity and neurologic outcomes. MIP images were generated from subtracted arterial phase images. Different projections by MPR techniques were used for optimal demonstration of the target vascular portion. Stenosis on MIP images was estimated by visual inspection, and the readers could adjust the window width and level if needed.

After PCO, the circle of Willis redistributes blood flow via the AcomA and the ipsilateral PcomA to the occluded side of the brain. The blood flows from the contralateral ICA, goes along the contralateral A1 segment and the AcomA in an anterograde manner and then along the ipsilateral A1 segment in a retrograde manner, eventually supplying the ipsilateral MCA territory. Meanwhile, the recruitment of blood flow from the basilar artery goes through the ipsilateral P1 segment and PcomA to supply the ipsilateral MCA territory.

The narrowest portions of the vessels were presumed to dominate the collateral blood flow. Both readers classified the narrowest portion of the AcomA and bilateral A1 segments (forming the anterior collateral vessels), which dominate the collateral flow from the contralateral ICA after PCO, into aplastic $(<25 \%$ of the ipsilateral A2 segment) (Fig 1A), hypoplastic (25\%-50\% of the 

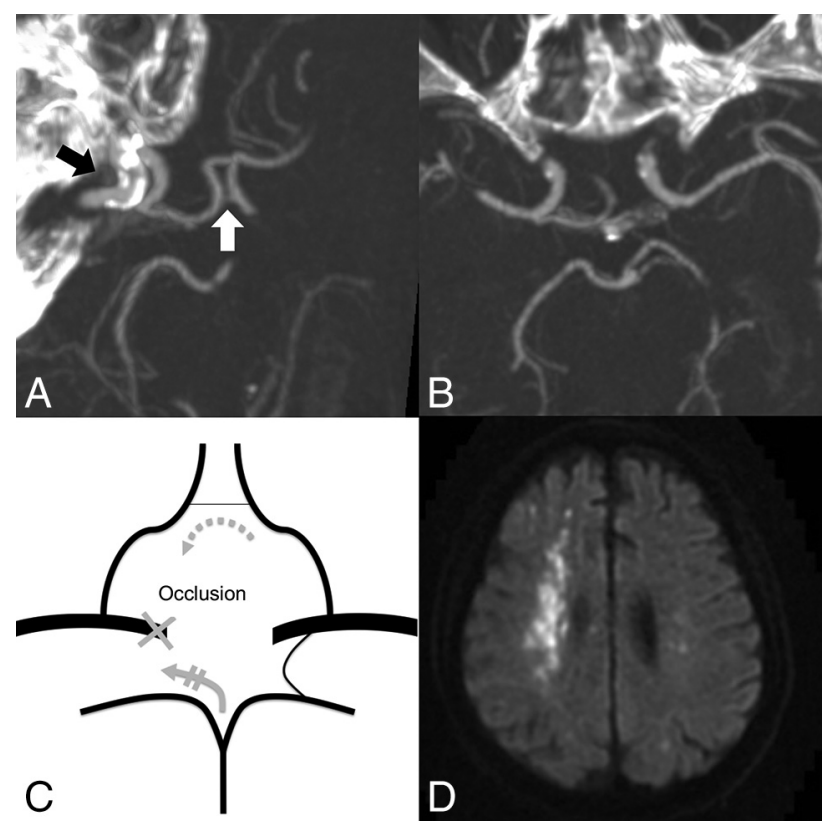

FIG 2. This 74-year-old man with nasopharyngeal carcinoma presented with massive hematemesis and epistaxis. A, Faint opacification of the AcomA (white arrow) is shown on the oblique MIP image ( 7 $\mathrm{mm}$ ), which is hypoplastic. The target of embolization is the right ICA (black arrow) due to an ICA pseudoaneurysm (not shown). B, No right PcomA is detected on the axial MIP image $(7 \mathrm{~mm})$. C, This patient is interpreted as high risk because only 1 hypoplastic AcomA supplies the collateral flow (gray arrows). D, Right borderzone infarction and tiny left embolic infarctions developed 5 days after right ICA embolization (shown on diffusion-weighted imaging).

ipsilateral A2 segment) (Fig $2 A$ ), and patent ( $>50 \%$ of the ipsilateral A2 segment) (Figs $3 A$ and $4 A$ ) segments. Likewise, the readers classified the narrowest portion of the ipsilateral PcomA and P1 segments (forming the posterior collateral vessels), which form the collateral flow from the basilar artery after PCO, into aplastic ( $<25 \%$ of the ipsilateral P2 segment) (Figs $1 B, 2 B$, and $3 B$ ), hypoplastic (25\%-50\% of the ipsilateral P2 segment), and patent (>50\% of ipsilateral P2 segment) (Fig 4B) segments. Steno-occlusive disease of the contralateral CCA and/or ICA and anatomic variants of the intracranial vasculature were also evaluated.

Both readers concluded their interpretation on collateral reserve, if disruption (ie, PCO) occurred, with a 5-point scale regarding the risk of borderzone infarction: very high risk, high risk, intermediate risk, low risk, and very low risk. If there was disagreement between the 2 readers, a consensus decision was made after discussion. In brief, assessment was as follows: The collateral reserve of the circle of Willis would be considered very high risk if both the anterior and posterior collateral vessels were aplastic (Fig 1), high risk if one was aplastic and the other was hypoplastic (Fig 2), intermediate risk if both were hypoplastic, low risk if one was patent and the other was aplastic (Fig 3), and very low risk if one was patent and the other was hypoplastic or patent (Fig 4).

\section{PCO Protocol}

Before every procedure, written informed consent was obtained from the patient and/or his or her family. All the PCO procedures were conducted in a biplane angiographic suite (Axiom Artis; Siemens, Erlangen, Germany). In our institution, PCO is the first-

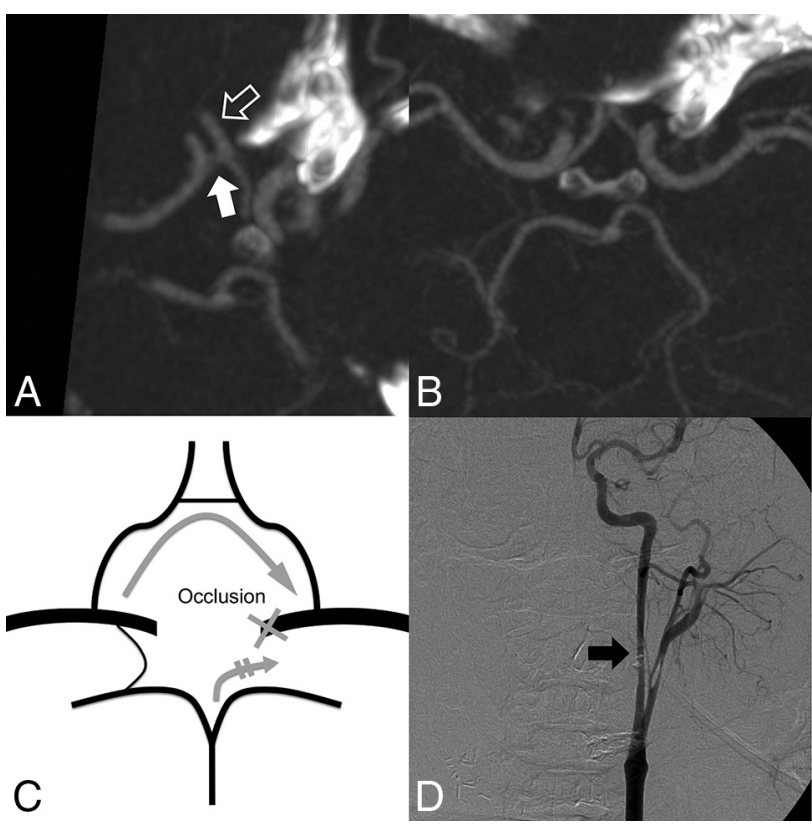

FIG 3. Images in a 36-year-old man with massive bleeding from a protruding tumor. A, Oblique MIP image $(7 \mathrm{~mm})$ shows that the AcomA (white arrow) diameter is $>50 \%$ of the ipsilateral A2 artery (PCO side, hollow arrow). B, The left PcomA on the axial MIP image (5 $\mathrm{mm}$ ) is aplastic. $C$, The patient is interpreted as low risk. The recruited collateral flows after PCO are indicated by gray arrows. D, Intraprocedural angiography shows irregular narrowing of the diseased left ICA (black arrow). No neurologic deficit was detected after left PCO.

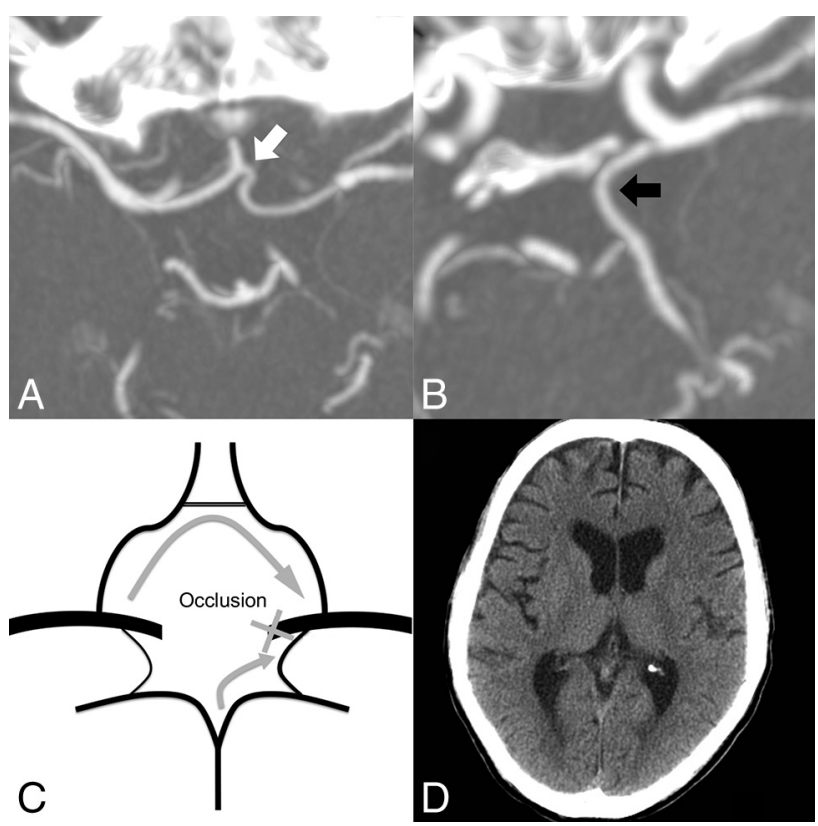

FIG 4. A 52-year-old man with hypopharyngeal cancer and bleeding from the exposed left carotid artery. A, Axial MIP image $(7 \mathrm{~mm})$ shows a well-opacified AcomA (white arrow), which is $>50 \%$ of the ipsilateral (PCO side) A2 segment (not shown). B, An axial MIP image (7 mm) reveals the left PcomA (black arrow), which is considered patent. $C$, The patient is considered very low risk. The recruited collateral flows after PCO are indicated by gray arrows. D, No cerebral infarction was found on brain CT 14 days after the left ICA embolization.

line treatment for CBS with pathologic lesions located in the CCA and/or ICA. To save time and to prevent excessive bleeding from anticoagulant use, we did not perform BTO before embolization. 
Likewise, we did not deploy stent grafts for CBS because longterm neurologic outcomes have not been shown to be superior to $\mathrm{PCO}^{9,16-18}$ and because poststenting antiplatelet and anticoagulant therapy is not preferred in CBS.

The timing of PCO was based on the clinical condition, CTA findings, and the willingness of the patient and/or family. Conservative managements, such as gauze packing, local compression, blood transfusion, fluid challenge, and/or tracheostomy were given if a patient refused PCO. The extent of PCO was planned according to the CTA images and correlated with angiography. The vertebral arteries and contralateral CCA were checked before the procedure ended. Platinum coils were used as the primary embolic agent, and vascular plugs were reserved for CCA occlusion. After PCO, we routinely elevated the systolic blood pressure to between 120 and $160 \mathrm{~mm} \mathrm{Hg}$ to ensure sufficient brain perfusion.

\section{Neurologic Outcomes}

After PCO, patients were admitted to an intensive care unit and monitored closely for signs of developing neurologic deficits, changing blood pressure, and rebleeding. The severity of the neurologic deficit was determined according to the summary of the neurology and rehabilitation consultation notes during admission. One radiologist (B.-C.L.) identified borderzone infarction and other thromboembolic infarction within 1 month after embolization by CT or MR imaging ${ }^{19}$; both were available for all patients with neurologic deficits. Borderzone infarction with extension to territorial regions was grouped as borderzone infarction. Patients were followed after the initial CTA to the last hospital visit or death.

\section{Statistical Analysis}

All statistical analyses were performed with MedCalc statistical software (Version 15.4.0.0; MedCalc Software, Mariakerke, Belgium). Reserve ratings of very high risk, high risk, and intermediate risk were considered positive, and collateral reserve ratings of low risk and very low risk were considered negative, to determine the sensitivity and specificity. Receiver operating characteristic (ROC) analysis was performed, and sensitivities and specificities were calculated for the performance of readers when predicting neurologic outcome after PCO. The diagnostic performance (areas under the ROC curve, $\left[\mathrm{A}_{\mathrm{z}}\right]$ ) of CTA for different neurologic outcomes was compared by paired comparison of the ROC curves using the DeLong method. ${ }^{20}$ The interobserver reliability was analyzed by the linear weighted- $\kappa$ statistic, with a value of $0.61-0.80$ indicating substantial agreement and a value of $0.81-1.00$ indicating excellent agreement. For all statistical analyses, a $P$ value $<.05$ was considered statistically significant.

\section{RESULTS}

The patients ( $n=31,4$ women and 27 men) enrolled in our study had a mean age of 53.7 years (range, 33-79 years). The primary diagnosis was nasopharyngeal carcinoma in $41.9 \%$ (13/31) of patients, oropharyngeal cancer in $19.4 \%(6 / 31)$, oral cancer in $12.9 \%(4 / 31)$, hypopharyngeal cancer in $19.4 \%(6 / 31)$, laryngeal cancer in $3.2 \%(1 / 31)$, and thyroid cancer in $3.2 \%(1 / 31)$. The clinical characteristics of these patients are shown in On-line
Table 1 . The quality of CTA was considered excellent in 28 patients $(90.3 \%, 28 / 31)$ and fair in the other 3 patients $(9.7 \%, 3 / 31)$. The fair ratings were because of the suboptimal timing of the arterial phase, which resulted in opacification of the cavernous sinus.

\section{Accuracy of CTA for Predicting Neurologic Outcome}

The stratified stroke risk in the cerebral collateral model is shown in the Table. All-cause stroke occurred in $10(32.3 \%, 10 / 31)$ patients, with the pattern being borderzone infarction in 7 patients, left retinal artery infarction in 1 patient, right retinal artery infarction in 1 patient, and right anterior cerebral artery infarction in 1 patient. The right anterior cerebral artery infarction was caused by inadvertent coil migration to the ipsilateral anterior cerebral artery.

The diagnostic performance and interobserver agreement of the CTA ratings are shown in On-line Table 2. For predicting borderzone infarction, the ROC curve analysis demonstrated $\mathrm{A}_{\mathrm{z}}$ values of $0.97,0.938$, and 0.914 for the experienced reader (C.-W.L.), the overall consensus, and the less-experienced reader (Y.-H.L.), respectively. No significant difference in the ROC curves was found between the 2 readers $(P=.114)$. Based on the consensus results, the sensitivity, specificity, positive predictive value, and negative predictive value of CTA for borderzone infarction were $100 \%$ (7/7), $62.5 \%$ (15/24), 43.8\% (7/16), and 100\% (15/15), respectively. Based on our findings, patients with either patent anterior or posterior collateral vessels (forming low-risk and very low-risk classifications) were thought to have adequate collateral reserve to prevent borderzone infarction after PCO. Interobserver agreement was substantial for anterior and posterior collateral vessels $(\kappa=0.631)$ and was excellent for the collateral reserve $(\kappa=0.807)$. The $\mathrm{A}_{\mathrm{z}}$ values of CTA were lower for all-cause stroke than for borderzone infarction for both readers and the consensus.

\section{Technical Outcomes}

Rebleeding episodes occurred in $5(16.1 \%, 5 / 31)$ patients between days 3 and 64 after the initial PCO, and 4 of them required further endovascular treatment. One patient did not undergo further angiography because compact gauze packing was effective for bleeding control. The rebleeding occurred in the contralateral CCA in one case because of advanced tumor involvement. Of the other 3 cases, rebleeding occurred in the ipsilateral external carotid artery in 2 cases and the ipsilateral vertebral artery in 1 case.

\section{Anatomic Variants of the Circle of Willis}

A fetal origin of the posterior cerebral artery was found in 3 patients (left in 2, right in 1). However, no persistent carotid-vertebrobasilar anastomosis was found in the studied patients.

\section{DISCUSSION}

In the present study, we demonstrated that CTA could be used to predict borderzone infarction after PCO in patients with CBS. Our results showed that 1 patent anterior or posterior collateral vessel, regardless of the integrity of the remaining circle of Willis, would be enough to prevent borderzone infarction after PCO. Moreover, the reader's experience had no prominent effect on the 
Assessment of the anterior and posterior collateral vessels and the collateral reserve

\begin{tabular}{|c|c|c|c|c|}
\hline & \multirow{2}{*}{$\begin{array}{c}\text { Collateral } \\
\text { Reserve }\end{array}$} & \multicolumn{3}{|c|}{ Anterior Collateral Vessels ${ }^{a}$} \\
\hline & & Patent & Hypoplastic & Aplastic \\
\hline Posterior collateral vessels ${ }^{\mathrm{b}}$ & $\begin{array}{l}\text { Patent } \\
\text { Hypoplastic } \\
\text { Aplastic }\end{array}$ & $\begin{array}{l}\text { Very low risk } \\
\text { Very low risk } \\
\text { Low risk }\end{array}$ & $\begin{array}{l}\text { Very low risk } \\
\text { Intermediate risk } \\
\text { High risk }\end{array}$ & $\begin{array}{l}\text { Low risk } \\
\text { High risk } \\
\text { Very high risk }\end{array}$ \\
\hline
\end{tabular}

${ }^{a}$ AcomA and bilateral A1.

I Ipsilateral PcomA and P1.

ROC analysis, implying that the result may be generalized to lessexperienced readers. This finding has not, to our knowledge, been reported previously, and it may expand to other conditions that require $\mathrm{PCO}$, such as carotid artery cavernous fistulas, inoperable giant carotid artery aneurysms, or carotid trunk lacerations.

The collateral potential of the circle of Willis, which allows alternative routes for the cerebral circulation when the cerebral blood supply is decreased, was first described by Sir Thomas Willis in $1664 .^{21}$ In patients undergoing PCO, diminished arterial pressure is expected at the postocclusive carotid artery, and neurologic manifestations can be highly variable; whereas some patients remain asymptomatic, others develop devastating borderzone infarctions in the deprived territory. ${ }^{9}$ The ability of the circle of Willis to recruit collaterals to the deprived part of the brain is crucial to stroke avoidance, similar to that seen with carotid disease caused by atherosclerotic occlusive disease. ${ }^{22,23}$ It has been shown that the existence of collateral flow from the PcomA was related to a lower incidence of borderzone infarctions in patients with unilateral ICA occlusion. ${ }^{11}$ However, if the collateral blood flow cannot meet the demand of the deprived territory, borderzone infarction will still occur. Several other factors, such as coil migration and possible cerebral thromboembolism during coil deployment, might contribute to the development of thromboembolic infarction after PCO. However, such factors are more related to the technical aspect of endovascular treatment itself and are embolic in nature, so they cannot be predicted by our model, as shown by the finding that the $A_{z}$ values of CTA were lower for all-cause stroke than for borderzone infarction.

BTO, which involves temporarily occluding the ICA under neurologic monitoring, is an established way of assessing the cerebral blood flow dependence on a particular ICA. ${ }^{12}$ In patients who pass the BTO, the incidence of neurologic complications is 3.0\%-6.7\% after permanent ICA occlusion. ${ }^{24}$ However, the total occlusion time needed for most BTO methods, which usually last 30-40 minutes, may not be tolerable for patients with $\mathrm{CBS},{ }^{25}$ and BTO was reported to have a high false-negative rate of $5 \%-20 \%$ in previous studies. ${ }^{26,27}$ Recently, venous phase BTO, which measures the discrepancy of venous filling between both hemispheres during ICA occlusion, has been introduced and requires only 60-90 seconds of occlusion time. ${ }^{13}$ Although bilateral femoral cannulation is needed for venous phase BTO, the low complication rate $(0 \%-0.7 \%)$ and its short examination time are promising. Another viable but less-studied method is transcranial Doppler sonography or contralateral carotid angiography with ipsilateral manual carotid compression, which can be performed within 1 minute. ${ }^{28}$ However, most patients with CBS might have a large bleeding wound on the neck, which would be thickly packed to stop bleeding, or postirradiation fibrosis, which would make manual carotid compression difficult and incomplete. BTO is still the criterion standard when evaluating cerebral collateral reserve under nonemergency clinical scenarios and can be considered during emergent PCO if venous phase BTO is used. Given the noninvasiveness and predictive value of CTA, our methods can serve as an initial risk-stratification tool during the preoperative evaluation, and BTO may be reserved for cases with undetermined results.

Carotid stent-graft deployment may preserve the cerebral perfusion in patients with CBS, potentially preventing borderzone infarction. However, we did not perform stent-graft deployment for CBS in a unilateral carotid artery because long-term neurologic outcome after stent placement in this setting has not been shown to be superior to PCO. ${ }^{9,16-18}$ The rates of stent-related complications, such as instent thrombosis, cerebral ischemia, and septic embolism, have been reported to approach $25 \%-50 \%$, with neurologic complication rates of $10 \%-27.8 \% .^{9,29}$ The unacceptably high poststenting complication rates could be attributable to inadequate antiplatelet therapy during clinical emergencies and contamination from the exposed artery. Therefore, we did not consider that routine stent-graft deployment was justified in patients with CBS, though it might be beneficial if both the initial CTA and confirmative BTO were considered risky for borderzone infarction. Further prospective studies are needed to evaluate the long-term outcomes of stent grafts in patients with insufficient collateral circulations.

The present study had some limitations. We did not account for other less-frequent collateral flow to the embolized carotid trunk, such as persistent embryonic vessels or ophthalmic anastomoses from the ipsilateral external carotid artery, which may supply the ICA in a retrograde manner after PCO. These unaccounted collateral flows may explain why some high-risk patients $(n=3)$ did not develop borderzone infarction. However, the ophthalmic anastomoses are usually tiny and no persistent embryonic vessel was found in this study. Therefore, we believe that theses unaccounted collaterals may only contribute to a small portion of brain perfusion after PCO. Another limitation was that evaluation of the stenotic portions of collateral vessels, which sometimes follow complex routes and form redundant loops, may be difficult using visual inspection by inexperienced readers. However, a vascular diameter of $>0.6 \mathrm{~mm}$ can be confidently identified by combining source images, MIP, and MPR, ${ }^{30}$ which allows stratification of collateral vessels using index A2 (mean, $2.4 \mathrm{~mm}$ ) and P2 (mean, 2.1 $\mathrm{mm}$ ) diameters. ${ }^{31,32}$ Also, readers only had to distinguish patent or nonpatent collateral vessels to obtain a $100 \%$ sensitivity for borderzone infarction according to our study results.

\section{CONCLUSIONS}

CTA was suitable for estimating the collateral reserve of the circle of Willis in patients with CBS, thereby predicting the occurrence of borderzone infarction after PCO. Further study is warranted to test whether this result can be generalized to other imaging modalities, such as MR angiography, or even become an alternative to BTO in other clinical scenarios.

\section{ACKNOWLEDGMENTS}

We thank Cheng-Li Lin for her help on statistical analysis. 
Disclosures: Adam Huang-UNRELATED: Patents (Planned, Pending or Issued): I have a patent related to removing bone from $\mathrm{CT}$ angiography, but that patent has not been licensed to any company and it has not created any income.

\section{REFERENCES}

1. Cohen J, Rad I. Contemporary management of carotid blowout. Curr Opin Otolaryngol Head Neck Surg 2004;12:110-15 CrossRef Medline

2. Chaloupka JC, Putman CM. Endovascular therapy for surgical diseases of the cranial base. Clin Plast Surg 1995;22:417-50 Medline

3. Pereira J, Phan T. Management of bleeding in patients with advanced cancer. Oncologist 2004;9:561-70 CrossRef Medline

4. Maran AG, Amin M, Wilson JA. Radical neck dissection: a 19-year experience. J Laryngol Otol 1989;103:760-64 CrossRef Medline

5. McDonald MW, Moore MG, Johnstone PA. Risk of carotid blowout after reirradiation of the head and neck: a systematic review. Int $J$ Radiat Oncol Biol Phys 2012;82:1083-89 CrossRef Medline

6. Chang FC, Luo CB, Lirng JF, et al. Evaluation of the outcomes of endovascular management for patients with head and neck cancers and associated carotid blowout syndrome of the external carotid artery. Clin Radiol 2013;68:e561-69 CrossRef Medline

7. Moore OS, Karlan M, Sigler L. Factors influencing the safety of carotid ligation. Am J Surg 1969;118:666-68 CrossRef Medline

8. Chaloupka JC, Putman CM, Citardi MJ, et al. Endovascular therapy for the carotid blowout syndrome in head and neck surgical patients: diagnostic and managerial considerations. AJNR Am J Neuroradiol 1996;17:843-52 Medline

9. Chang FC, Luo CB, Lirng JF, et al. Endovascular management of post-irradiated carotid blowout syndrome. PLoS One 2015;10: e0139821 CrossRef Medline

10. Kulik T, Kusano Y, Aronhime S, et al. Regulation of cerebral vasculature in normal and ischemic brain. Neuropharmacology 2008;55: 281-88 CrossRef Medline

11. Hendrikse J, Hartkamp MJ, Hillen B, et al. Collateral ability of the circle of Willis in patients with unilateral internal carotid artery occlusion: border zone infarcts and clinical symptoms. Stroke 2001; 32:2768-73 CrossRef Medline

12. Mathis JM, Barr JD, Jungreis CA, et al. Temporary balloon test occlusion of the internal carotid artery: experience in $\mathbf{5 0 0}$ cases. AJNR Am J Neuroradiol 1995;16:749-54 Medline

13. Sorteberg A. Balloon occlusion tests and therapeutic vessel occlusions revisited: when, when not, and how. AJNR Am J Neuroradiol 2014;35:862-65 CrossRef Medline

14. Lee CW, Yang CY, Chen YF, et al. CT angiography findings in carotid blowout syndrome and its role as a predictor of 1-year survival. AJNR Am J Neuroradiol 2014;35:562-67 CrossRef Medline

15. Katz DA, Marks MP, Napel SA, et al. Circle of Willis: evaluation with spiral CT angiography, MR angiography, and conventional angiography. Radiology 1995;195:445-49 CrossRef Medline

16. Simental A, Johnson JT, Horowitz M. Delayed complications of endovascular stenting for carotid blowout. Am J Otolaryngol 2003;24: 417-19 CrossRef Medline

17. Kim HS, Lee DH, Kim HJ, et al. Life-threatening common carotid artery blowout: rescue treatment with a newly designed self-expanding covered nitinol stent. Br J Radiol 2006;79:226-31 CrossRef Medline

18. Gaba RC, West DL, Bui JT, et al. Covered stent treatment of carotid blowout syndrome. Semin Intervent Radiol 2007;24:47-52 CrossRef Medline

19. Mangla R, Kolar B, Almast J, et al. Border zone infarcts: pathophysiologic and imaging characteristics. Radiographics 2011;31:1201-14 CrossRef Medline

20. DeLong ER, DeLong DM, Clarke-Pearson DL. Comparing the areas under two or more correlated receiver operating characteristic curves: a nonparametric approach. Biometrics 1988;44:837-45 CrossRef Medline

21. Eastcott HH. The beginning of stroke prevention by surgery. Cardiovasc Surg 1994;2:164-69 Medline

22. Klijn CJ, Kappelle LJ, Tulleken CA, et al. Symptomatic carotid artery occlusion: a reappraisal of hemodynamic factors. Stroke 1997;28: 2084-93 CrossRef Medline

23. Lima FO, Furie KL, Silva GS, et al. The pattern of leptomeningeal collaterals on CT angiography is a strong predictor of long-term functional outcome in stroke patients with large vessel intracranial occlusion. Stroke 2010;41:2316-22 CrossRef Medline

24. American Society of Interventional and Therapeutic Neuroradiology. Carotid artery balloon test occlusion. AJNR Am J Neuroradiol 2001; 22:S8-9 Medline

25. Zussman B, Gonzalez LF, Dumont A, et al. Endovascular management of carotid blowout. World Neurosurg 2012;78:109-14 CrossRef Medline

26. Marshall RS, Lazar RM, Young WL, et al. Clinical utility of quantitative cerebral blood flow measurements during internal carotid artery test occlusions. Neurosurgery 2002;50:996-1004; discussion 1004-05 Medline

27. Schneweis S, Urbach H, Solymosi L, et al. Preoperative risk assessment for carotid occlusion by transcranial Doppler ultrasound. J Neurol Neurosurg Psychiatry 1997;62:485-89 CrossRef Medline

28. Hetzel A, von Reutern G, Wernz MG, et al. The carotid compression test for therapeutic occlusion of the internal carotid artery: comparison of angiography with transcranial Doppler sonography. Cerebrovasc Dis 2000;10:194-99 CrossRef Medline

29. Hakime A, Khoury E, Hameg A, et al. Polytetrafluoroethylene-covered nitinol stent graft for treatment of carotid artery blowout syndrome in head and neck cancer patients. Laryngoscope 2013;123: 1670-75 CrossRef Medline

30. Skutta B, Fürst G, Eilers J, et al. Intracranial stenoocclusive disease: double-detector helical CT angiography versus digital subtraction angiography. AJNR Am J Neuroradiol 1999;20:791-99 Medline

31. Kawashima M, Rhoton AL Jr, Tanriover N, et al. Microsurgical anatomy of cerebral revascularization, Part II: posterior circulation. J Neurosurg 2005;102:132-47 CrossRef Medline

32. Kawashima M, Rhoton AL Jr, Tanriover N, et al. Microsurgical anatomy of cerebral revascularization, Part I: anterior circulation. J Neurosurg 2005;102:116-31 CrossRef Medline 